Madrygal. Revista de Estudios Gallegos

ISSN: 1138-9664

\title{
¿Absoluta imposibilidad o errores de otros tiempos? Emilia Pardo Bazán y Prudencio Canitrot
}

\author{
Carlos Gegúndez López ${ }^{1}$
}

Recibido: 3 de setembro de 2016 / Aceptado: 22 de outubro de 2016

Resumen. Este artículo reflexiona sobre la relación entre Emilia Pardo Bazán y Prudencio Canitrot. Estos dos escritores gallegos mantuvieron contacto en Galicia y Madrid, compartieron momentos de cordialidad, aunque también se han observado algunas diferencias ideológicas y tensiones en esa relación, aspectos que analizamos en el presente artículo. Palabras clave: Emilia Pardo Bazán; Prudencio Canitrot; biografía; relaciones; discrepancias.

\section{[gl] Absoluta imposibilidade ou erros doutros tempos? Emilia Pardo Bazán e Pru- dencio Canitrot}

Resumo. Este artigo reflexiona sobre a relación entre Emilia Pardo Bazán e Prudencio Canitrot. Estes dous escritores galegos mantiveron contacto en Galicia e Madrid, compartiron momentos de cordialidade, aínda que tamén se teñen observado algunhas diferenzas ideolóxicas e tensións nesa relación, aspectos que analizamos no presente artigo.

Palabras chave: Emilia Pardo Bazán; Prudencio Canitrot; biografía; relacións; discrepancias.

\section{[en] An Absolute Impossibility or a Mistake from the Past? Emilia Pardo Bazán and Prudencio Canitrot}

Abstract. This article reflects on the relationship between Emilia Pardo Bazán and Prudencio Canitrot. Although ideological differences and tensions between them are evident, both writers were close friends who kept in contact in Galicia and Madrid.

Keywords: Emilia Pardo Bazán; Prudencio Canitrot; Biography; Relationships; Disagrees.

Sumario. 1. Madrid y Galicia como puntos de encuentro. 2. Los "errores" de doña Emilia. 3. La "absoluta imposibilidad" de Pardo Bazán. 4. Referencias bibliográficas.

Como citar: Gegúndez López, C. (2016): “¿Absoluta imposibilidad o errores de otros tiempos? Emilia Pardo Bazán y Prudencio Canitrot”, en Madrygal. Revista de Estudios Gallegos 19, 71-78.

\footnotetext{
Universidade da Coruña, Facultade de Ciencias da Educación, Departamento de Didácticas Específicas.
}

E-mail: carlos.gegundez.lopez@udc.es 


\section{Madrid y Galicia como puntos de encuentro}

No hemos podido documentar con absoluta precisión en qué momento se conocieron Emilia Pardo Bazán y Prudencio Canitrot, "joven escritor de la cuerda de Valle-Inclán" (Cansinos 1995: 454), aunque lo que sí parece claro es que ese primer encuentro se produjo en Madrid, ciudad en la que se instaló el autor pontevedrés a comienzos del siglo XX. La relación entre estos dos escritores empezó en la capital del Estado y tuvo algunos momentos de tensión y otros de distensión, tal y como expondremos en este texto. Como ya hemos dicho, no podemos fijar la fecha concreta de ese primer encuentro, aunque sabemos con certeza que se produjo entre los años 1906 y 1908. Por aquel entonces, el joven escritor gallego ya se había ido distanciando de las excéntricas tertulias bohemias y empezaba a entablar relación con otros periodistas y escritores en el Ateneo. Un ambiente intelectual, formal, donde la excentricidad no tenía cabida, ya que como señaló un autor gallego: "Ser ateneísta era daquela a aposta intelectual seria e discreta, fóra das viaxes dolorosas pola noite literaria da corte" (Fonte 1985: 33). Es posible que esa primera toma de contacto entre ambos autores se produjese en el Ateneo de Madrid. Independientemente del momento y lugar exacto en el que se conocen, lo verdaderamente importante en la tarea que nos ocupa es dejar claro que tras esa primera toma de contacto $\mathrm{u}$ otras anteriores que se pudiesen producir, en Madrid, antes del estío de 1908, Canitrot y Pardo Bazán entablaron una relación cordial, incluso amistosa; así lo demuestra un texto publicado por el escritor que apareció por primera vez en la revista Galicia, editada por el Centro Gallego de La Habana, que versa sobre una visita de Prudencio a la autora cuando ésta se hallaba en Galicia. En este caso, podemos constatar que se trata de la primera cita entre ambos en suelo gallego y tiene lugar durante el verano del año 1908. Probablemente fuese también la última, si excluimos otras que pudiesen producirse fruto de la casualidad. Además, resulta bastante improbable que esos hipotéticos contactos posteriores, en el caso de haber tenido lugar, discurriesen en el mismo tono y con la misma cordialidad mostrada por ambos durante el primer encuentro. El mencionado texto publicado por Prudencio en Galicia, solo unos meses después de su visita a la Condesa así lo constata. Un encuentro que se produce en este

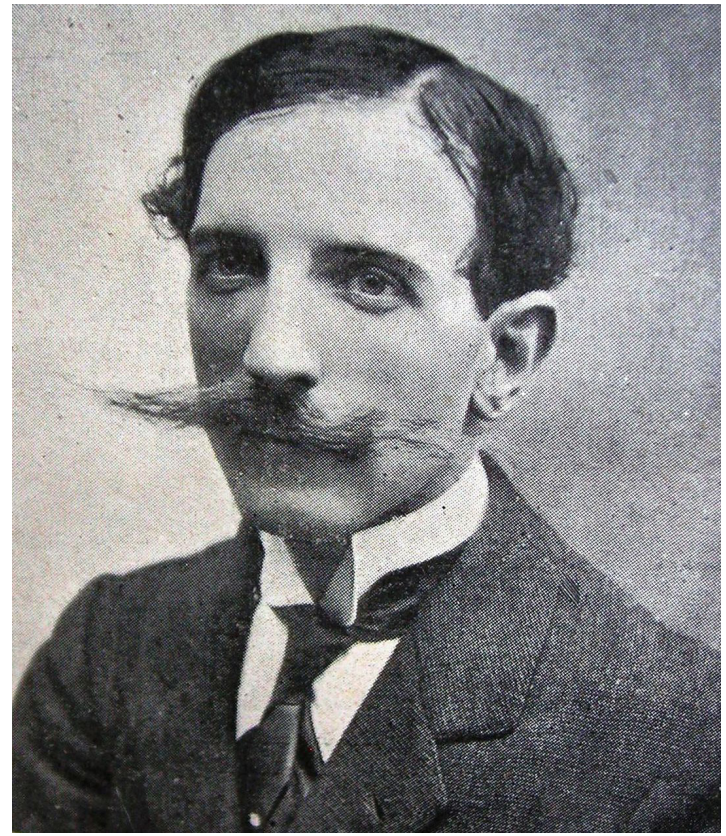

Fig. 1. Fotografía de Prudencio Canitrot (La luz apagada)

instante, aunque ya había sido concertado con bastante antelación:

Varias veces me había dicho en Madrid la ilustre escritora condesa de Pardo Bazán, que no dejase de visitarla en su palacio de Meirás cuando fuese á la Coruña, y unos días llevaba en esta población cuando una tarde decidí cumplir aquel su deseo y mi promesa. (Canitrot 1908a: 1)

Siguiendo el criterio autorial, se puede calificar el texto en el que relata dicho encuentro como una crónica de viaje. De hecho, el autor la integraría en su libro Rías de ensueño, un conjunto de textos en el que describe su recorrido por algunos parajes de las rías gallegas. En este caso, retrata el paisaje que observa mientras recorre el itinerario entre A Coruña y el Pazo de Meirás. Al llegar al lugar de residencia de la autora, Prudencio comprueba que ésta ha salido y decide esperarla hasta su regreso:

El débil sonido de unas colleras llega á mis oídos, abstrayéndome de la grata contemplación. Un coche rueda por la calle de magnolios y se detiene junto á la plazoleta, descendiendo de él doña Emilia y sus hijas. Me acerco á saludarlas, $\mathrm{y}$ en mis palabras hay una gran alabanza para el paisaje y para la gran escritora, que experimenta un profundo disgusto por no haber regresado antes de Avegondo y por no conocer de antemano mi visita. Yo prometo más oportunidad otro día, y un previo aviso. Consulto la hora, y echo de ver 
que me queda muy poco tiempo para regresar á Santa Cruz, y luego atravesar la bahía. Sin embargo, me queda tiempo para saborear una taza de te y la charla ingeniosa, elevada, á veces mordaz y otras despectiva, de la gran novelista. (Canitrot 1908a: 2)

Lo que nos interesa más allá de la tipología textual es fijar la atención en la actitud mostrada por ambos. Y si ponemos el foco en esta cuestión, podemos llegar a la conclusión de que la cordialidad entre estos dos autores es absoluta. El texto se publica por primera vez como ya hemos señalado en 1908 y el autor decide no modificarlo cuando decide incluirlo en un libro solo dos años más tarde (cf. Canitrot 1910: 155-162). Un aspecto muy significativo, más todavía si tenemos en cuenta que solo un año antes Canitrot había emitido algunos juicios contrarios a la ideología conservadora de Emilia Pardo Bazán y otros poco generosos para con la obra literaria de Emilia Pardo Bazán, tal y como veremos en el siguiente apartado de este artículo, por lo que se puede concluir que Prudencio siempre respetó a la escritora coruñesa. A finales de 1908 cuando Basilio Álvarez asume la dirección de la revista Galicia del Centro Gallego de Madrid nombrando a Prudencio Canitrot director artístico, la publicación anuncia una serie de cambios y mejoras entre las que se encuentra su deseo de contar con firmas de prestigio como la de Pardo Bazán. Y en 1910, al fundar la Biblioteca de Escritores Gallegos ${ }^{2}$, Canitrot y Luís Antón del Olmet anuncian la colaboración de autores destacados, entre los que incluyen el nombre de doña Emilia, aunque finalmente no aparecería ningún trabajo de su autoría ni en la revista ni en la colección literaria fundada por Canitrot.

Al margen de estas conjeturas y analizando la literatura de estos autores, algunos críticos de la época llegaron a establecer similitudes entre la obra literaria de ambos escritores, aunque esos paralelismos tienen más que ver con los escenarios y ambientes en los que discurren las historias de sus narraciones que con cuestiones propiamente estilísticas. De hecho, algún crítico contrapone con claridad la obra de ambos, situando a Canitrot al lado de Valle-Inclán y a doña Emilia a la cabeza de una "tendencia literaria" completamente diferenciada:
Entre los escritores gallegos representa, así, Fernández Flórez, en cuanto al estilo la tendencia más libre y moderna. Se pensaría al leer las novelas como Volvoreta y como El demonio de la vouptusidad de Alberto Insúa y como La Casa de la Troya de Lugín; y al compararlas con otras novelas regionales de Valle Inclán, Canitrot, etc., que hay dos Galicias contrapuestas, una sombría, supersticiosa, bárbara, y otra clara, sencilla, humana y moderna. Pero, sin duda lo que ocurre es que hay dos tendencias literarias; una que parte de Emilia Pardo Bazán y mantiene las buenas tradiciones de naturalidad y de verdad de la novela grande, y otra que tiene su arranque en Valle Inclán y exagera por un afán de preciosismo, las categorías regionales derivando hacia las formas novelescas menores, la leyenda y el poema. Entre una tendencia y la otra está el 1900. (Cansinos 1916: 3)

Las diferencias estilísticas entre la obra creativa de ambos son más que notables; seguramente tampoco contribuyó a que se establecieran este tipo de paralelismos un retrato del autor que Antonio Rey Soto trazó con su pluma. El escritor gallego describe a Prudencio como si se tratase de un personaje novelesco de doña Emilia:

¿Recordáis aquel amable, desenfadado, nervio-
so y espiritual personaje, semi-real y semi-
soñado, que nuestro primer novelista-la Pardo
Bazán- inmortalizó con el nombre de Silvio
Lago? Pues muy semejante á él en lo físico, y
tocado también del aliento de La Quimera, que
enloquece y mata. (Rey 1910: 7-8)

Curiosamente, la autora se inspiró en Vaamonde, un pintor gallego que la retrató en varias ocasiones, para crear a Silvio Lago, protagonista de La Quimera. Joaquín Vaamonde Cornide es uno de los malogrados artistas gallegos que fallece alrededor del año 1900, tal y como les sucede a Ovidio Murguía, Jenaro Carrero, Ramón Parada Justel y Jenaro Pérez Villamil. Todos ellos pasarían a integrar posteriormente el grupo que el pintor ferrolano Felipe Bello Piñeiro denominó "Generación Doliente". El retrato literario de Rey Soto acabaría siendo premonitorio, puesto que La Quimera de Pardo Bazán, "narra el amargo destino de un pintor fracasado -Joaquín Vaamonde, íntimo amigo de la condesa, muerto en plena juventud" 
(Latorre 1993-94: 503). Canitrot tendrá el mismo sino. Es como si el prologuista, adivinase por aquel entonces el destino final del escritor. En 1913, tres años después de la publicación de Ruinas, la citada obra prologada por Antonio Rey Soto, aquel joven que había soñado con ser pintor en un primer momento y que posteriormente se centró más en la escritura, cuando ya su firma empezaba a rubricar crónicas y artículos en destacadas publicaciones de la época y habiendo publicado media docena de obras de creación, con treinta años recién cumplidos, sería derrotado por la mortal tisis. Tal y como le había sucedido a Silvio Lago, la tuberculosis acabó con su vida.

\section{Los "errores" de doña Emilia}

Durante el invierno del año 1909, un grupo de intelectuales gallegos se reúne en Madrid y a la cabeza de todos ellos aparece Basilio Álvarez, quien trata de poner en marcha un ambicioso proyecto político que le permita ponerse al frente del movimiento agrarista en Galicia. $\mathrm{Su}$ aspiración es la misma que tienen otras organizaciones operantes dentro del agrarismo, la abolición de los contratos forales. El abad de Beiro se rodea de jóvenes escritores, entre ellos aparece Prudencio Canitrot, que forma parte del grupo que funda Acción Gallega. A comienzos de 1910 esta nueva organización política aparece en escena con una publicación quincenal que lleva el mismo nombre y en la que todos estos intelectuales ponen su pluma al servicio del grupo agrarista. Además, Álvarez logra atraer a Manuel Portela Valladares y a Alfredo Vicenti. Ambos políticos cuentan en ese momento con un destacado nombre en la política española y pasarán a integrar la candidatura de Acción Gallega por los distritos electorales de A Fonsagrada y Becerreá, respectivamente, en las Elecciones Generales de $1910^{3}$.

Si bien el compromiso de Canitrot con la causa agrarista es anterior a su militancia y activismo dentro de la nueva fuerza política, así lo demuestran un par de artículos publicados entre 1908 y 1909. Precisamente, en uno de ellos se refleja de forma manifiesta un desencuentro ideológico con Emilia Pardo Bazán. En estos textos sobre la cuestión foral el autor gallego muestra su preocupación por la situación del campesinado sometido a duras cargas impositivas, pero también manifiesta cierto optimismo ante los conatos de rebeldía de algunos campesinos, "...en donde aún perduran privilegios tan odiosos como todas las supervivencias abadengas que revisten verdadero carácter de gebla, corre un reguero de rebeldía, como corre el fuego por un campo maduro" (Canitrot 1909: 1).

En estos momentos el movimiento agrario alcanza uno de sus puntos álgidos. Además de la toma de conciencia por parte del campesinado que empieza a movilizarse, van entrando en escena varios actores políticos entre los que también se encuentran Solidaridad Gallega ${ }^{4}$, el Directorio de Teis o Unión Campesina. Algunos diputados gallegos como los citados Vicenti y Manuel Portela Valladares llevan propuestas de redención al Congreso de los Diputados, el propio Portela solicita en el Congreso al Marqués de Figueroa, Ministro de Gracia y Justicia la aplicación de la Ley de redención forzosa (cf. Durán 1977: 320). Ante esta situación tan reivindicativa, las clases arrendatarias ven amenazada su posición y reaccionan con firmeza. Elaboran un documento conjunto mediante el que expresan su oposición frontal a cualquier movimiento o rebelión social que pueda poner en peligro su posición de privilegio, tal y como señala un investigador: "El documento, apenas aparecer, resultó explosivo, más que por efecto de cerrar el camino parlamentario de la proposición Vicenti, por el estilo y calidad de los firmantes. Todas las grandes casas de Galicia, sus abolengos están allí" (ibid. 325). Además, algunos periódicos de la época, principalmente los afines a la ideología conservadora, advierten del tenso clima de agitación campesina que se vive en Galicia y de sus "nefastas consecuencias". Por esta razón, Canitrot escribe:

Portela Valladares lograría el acta por A Fonsagrada, pero el liberal Alfredo Vicenti no pudo lograr la suya por el distrito becerrense ante el candidato conservador Goicoechea.

4 Canitrot comparte las reivindicaciones de Solidaridad Gallega, pero cree que su labor es infructuosa: “Así es que la labor de los solidarios del campo, es sólo un paréntesis que se abre en Domingo, en el sufrido corazón del labriego, sahumándolo brevemente. Pero nada más." (Canitrot 1908b: 1). 
Y si por esto no fuera bastante, ahora, los hidalgos gallegos protestan también en lucha abierta contra la redención de foros, y unidos á ellos, ciertos personajes que nada tienen que ver con aquella tierra sin ventura, se asocian para luchar en la grave contienda que se avecina. (Canitrot 1909: 1)

Emilia Pardo Bazán, partidaria del mantenimiento del sistema de propiedad establecido, se manifiesta a favor de los rentistas y se adhiere a los firmantes del documento. Esta decisión provoca un gran malestar en el autor pontevedrés, que lamenta y critica con firmeza la posición de la autora:

Nuestra gran escritora Emilia Pardo Bazán, condesa y forataria, se pone al parecer á la cabeza de este movimiento. Poco se lo agradecerán sus paisanos que conocen bien su asombrosa labor literaria y reconocieron siempre que su pluma serena no hizo brotar nunca una lágrima, porque se seca como un roble de vetusto Señorío. (ibid.)

No se puede precisar con exactitud si Canitrot trata de rebajar su crítica al final del artículo, si bien reconoce su admiración por doña Emilia, y aunque se podría pensar que esas diferencias pudiesen ser exclusivamente ideológicas, acaba concluyendo: "Yo profeso á mi ilustre paisana una gran admiración; pero no olvido aquellos sus errores políticos de otros tiempos y los literarios de los tiempos presentes" (ibid.). Estos juicios emitidos por el autor solamente unos meses después del cordial encuentro en Meirás, pudieron molestar a la autora coruñesa e incluso es muy probable que provocasen un distanciamiento entre ambos y un enfriamiento de sus relaciones.

\section{La "absoluta imposibilidad" de Emilia Pardo Bazán}

Tras el fallecimiento de Prudencio Canitrot Mariño en 1913 y coincidiendo con el primer aniversario de su muerte, Isidoro Canitrot, hermano del autor decidió publicar un volumen póstumo con algunos de sus textos inéditos, rescatando además otros que no habían aparecido en libro con anterioridad, aunque la verdadera vocación de esta obra es homenajear al autor. Lleva por título La luz apagada y apareció a comienzos de 19145. Está dividida en varios apartados. Los tres primeros recogen cuentos, crónicas y "prosas iluminadas" (prosas líricas). Todos ellos, son textos escritos por el autor homenajeado. El cuarto y último capítulo se titula "artículos necrológicos" y recopila una serie de textos elegíacos de homenaje al autor. Las colaboraciones se corresponden con una destacada nómina de intelectuales que mantuvieron amistad o conocieron al escritor pontevedrés ${ }^{6}$. Para prologar este libro, no sabemos con certeza si fue el coordinador de la edición, Isidoro Canitrot o la viuda del autor, Marina de la Arena Pérez de Vargas quien le hizo el ofrecimiento a Emilia Pardo Bazán. Tenemos constancia de que fue Pérez de Vargas quien realizó la petición por carta, aunque la autora coruñesa acabaría remitiendo su respuesta negativa al hermano del autor. Doña Emilia declina el encargo a través de una carta fechada el 12 de diciembre de 1913, justificando (Fig. 2):

Muy Sr. Mío y de todo mi aprecio:

$\mathrm{Su}$ Sra. hermana política, la Sra. viuda de Canitrot, me ha escrito solicitando un prólogo para una obra de su hermano, que está próximo á publicarse.

Al recibir la carta, no me fijé en que no traía señas; hoy la repaso, y no habiendo podido averiguar en El Liberal las señas de esta señora, me han aconsejado que me dirija á Vd., que fácilmente hará llegar hasta ella su respuesta.

Habiendo admirado como se merecía el talento de su malogrado hermano, sin duda prologaría con sumo gusto su obra póstuma, si no me lo impidiese la absoluta imposibilidad en que me encuentro de distraer un minuto, en algunos

\footnotetext{
En una nota inicial que introduce la obra se puede leer: "Hemos querido reunir en estas páginas efusivas y cordiales, el cariño y la admiración que nos inspiraba el amigo muerto. De cordialidad y de efusión fueron siempre sus obras. Vibraba con ellas la luz interior y sonaba la voz dulce, cadenciosa de la tierra que le vió nacer y no ha sido su sepultura. El recuerdo de aquella luz, hoy apagada, y de aquella voz que enmudeció para siempre es lo que ofrecemos como un ex voto, como una ofrendaa".

$6 \quad$ Este último apartado que corona el volumen contiene textos en prosa y verso en homenaje a Prudencio Canitrot colaboran Alfredo Vicenti, Salvador Rueda, Cristóbal de Castro, Sofía Casanova, José Francés, Antonio Palomero, Alberto Insúa, Celso Lucio, Luis Antón del Olmet, El Marqués de Figueroa, Manuel Murguía, Javier Valcarce, Federico Gil Asensio, Pedro Penzol, Augusto Martínez Olmedilla, Victoriano García Martí, Emma Calderón y de Gálvez, Juan Barcia Caballero, Francisco Tettamancy, Basilio Álvarez y el propio Isidoro Canitrot Mariño.
} 
meses, de los trabajos apremiantes prometidos á editores, cuyos intereses sufrirían con el incumplimiento de mi compromiso.

Mucho siento no poder complacer á esa Señora, á quien ruego salude $\mathrm{Vd}$. en mi nombre quedando suya afectísima, La Condesa de Pardo Bazán. (Gegúndez 2014: 344)7

Solo unos días más tarde, Manuel Linares Rivas le escribe una misiva a Isidoro Canitrot ofreciendo su absoluta disposición a colaborar en La luz apagada. Seguramente el hermano del autor fallecido y ante la negativa de Pardo
Bazán, le ofreció la posibilidad de escribir el prólogo tal como se deduce en la carta remitida por Linares Rivas el 17 de diciembre de 1913:

\section{Sr. Dr. Canitrot}

Muy seño mío: tendré a mucha honra unir mi firma al libro del malogrado Prudencio. Dígame si lo que desea es un artículo encomiástico ó un trabajo de distinta índole; en una palabra, si quiere usted algo a manera de prólogo, o una composición libre para formar volumen.

Con este motivo me ofrezco muy gustoso, su amigo q.1.s. Manuel Linares Rivas. (Fig. 3) ${ }^{8}$

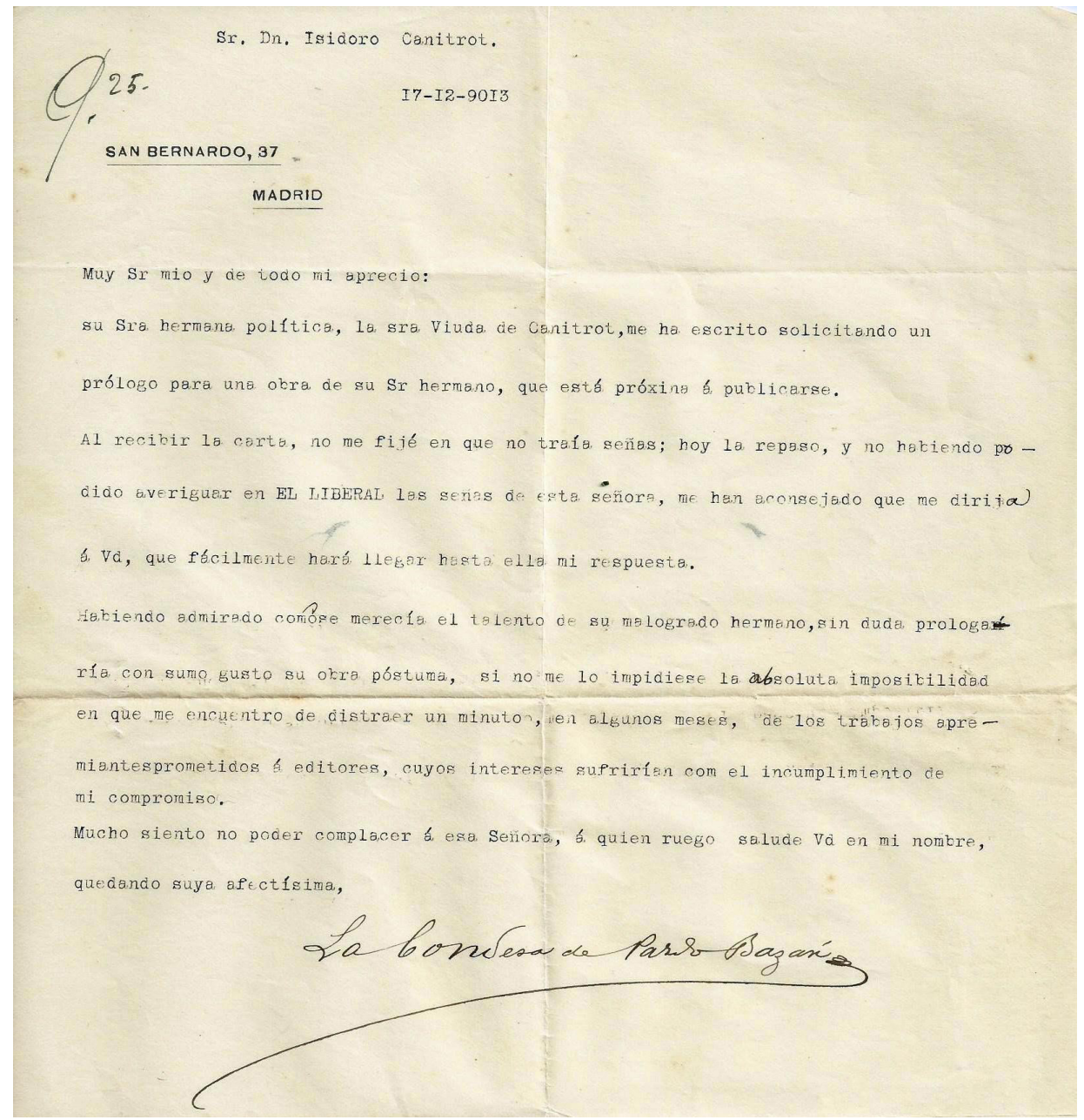

Fig. 2. Carta mecanografiada de Emilia Pardo Bazán a Isidoro Canitrot (Archivo de María de los Ángeles Posse Canitrot)

Transcripción de una carta remitida por Emilia Pardo Bazán a Prudencio Canitrot perteneciente al archivo de María de los Ángeles Posse Canitrot a quien agradecemos su colaboración para la redacción de este artículo. La carta aparece recopilada en la tesis doctoral de Carlos Gegúndez López, Canitrot e o sistema literario galego no tránsito de século. Un proxecto didáctico entre o humanismo e a estética galega (2014) y que aparece publicada por primera vez en el presente número de Madrygal.

8 Carta remitida por Manuel Linares Rivas a Isidoro Canitrot, al igual que la carta de Doña Emilia, forma parte del archivo de María de los Ángeles Posse Canitrot y aparece recopilada en la mencionada tesis de Gegúndez López. 


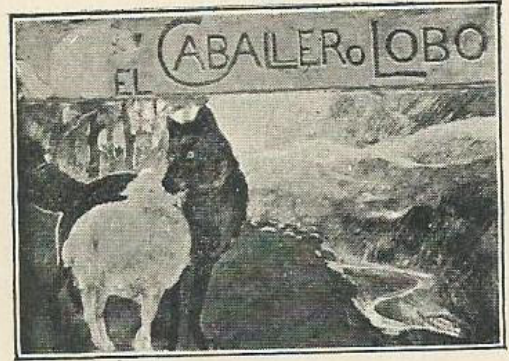

\section{Atmaram}
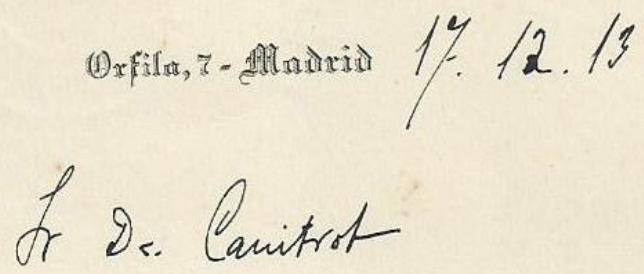

hun veñor nio: tendré a muche honra el mir mi firma

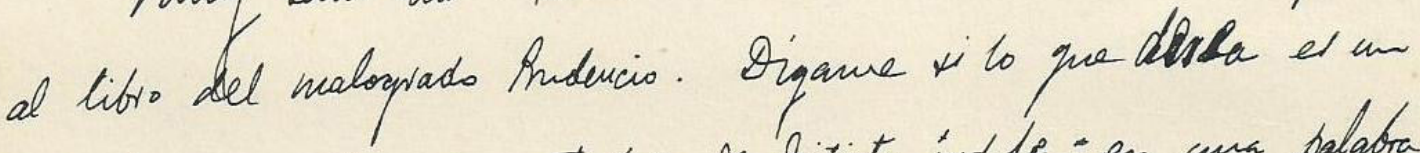
articulo encomiartico ó un trabajs be ristiuto údole; en cura palabra ; quiere t. algo a manera de prólogo, o una comporicion libie para formuer columen.

Fig. 3. Carta manuscrita de Manuel Linares Rivas a Isidoro Canitrot

(Archivo de María de los Ángeles Posse Canitrot)

Finalmente, sería el abogado y escritor coruñés el autor del prólogo escrito el 6 de enero de 1914. Este encargo le permitió cumplir con una petición que le había hecho el propio Prudencio en vida. El escritor pontevedrés le había solicitado un prólogo para un libro que no se llegaría a publicar a causa de su fallecimiento. Por esta razón Linares señala en el prefacio de La luz apagada: “...y acudieron á mí para que yo escribiera el prólogo de este epitafio literario. Y al preguntarme: ¿quiere...?, contesté: sí, hombre: como si otra vez y nuevamente respondiera á la misma invitación..." (Linares 1914: 9).

Volviendo sobre la negativa de Emilia Pardo Bazán y a modo de conclusión debemos reconocer de forma indudable que esas ocupaciones de las que habla la autora de $\operatorname{Los} P a$ zos de Ulloa no eran seguramente cuestiones menores, aunque también cuesta creer que no pudiese disponer de tiempo para escribir el prólogo que le habían encargado, ni siquiera para enviar un pequeño texto que se pudiese insertar en la corona final del libro y que va encabezada con el título "Artículos necrológicos". Si bien esas ocupaciones editoriales pudiesen ser una justificación para no escribir el texto, creemos que doña Emilia no tenía la mejor disposición para poner su pluma en esta obra. Por tanto, no podemos afirmar de forma absolutamente categórica que use su carga de trabajo como excusa o pretexto para no intervenir, pero sorprende que la escritora rechazase participar en La luz apagada, ni siquiera con un pequeño texto en homenaje. Es indudable que nunca sabremos con certeza la verdadera razón por la que declinó prologar la obra, pero parece claro su desinterés por tomar parte en este libro. Por todo ello, pensamos que no fue únicamente la "absoluta imposibilidad" de la autora lo que se lo impidió y es más que probable que también pesasen mucho aquellos "errores de otros tiempos" de los que había hablado Canitrot en el pasado. 


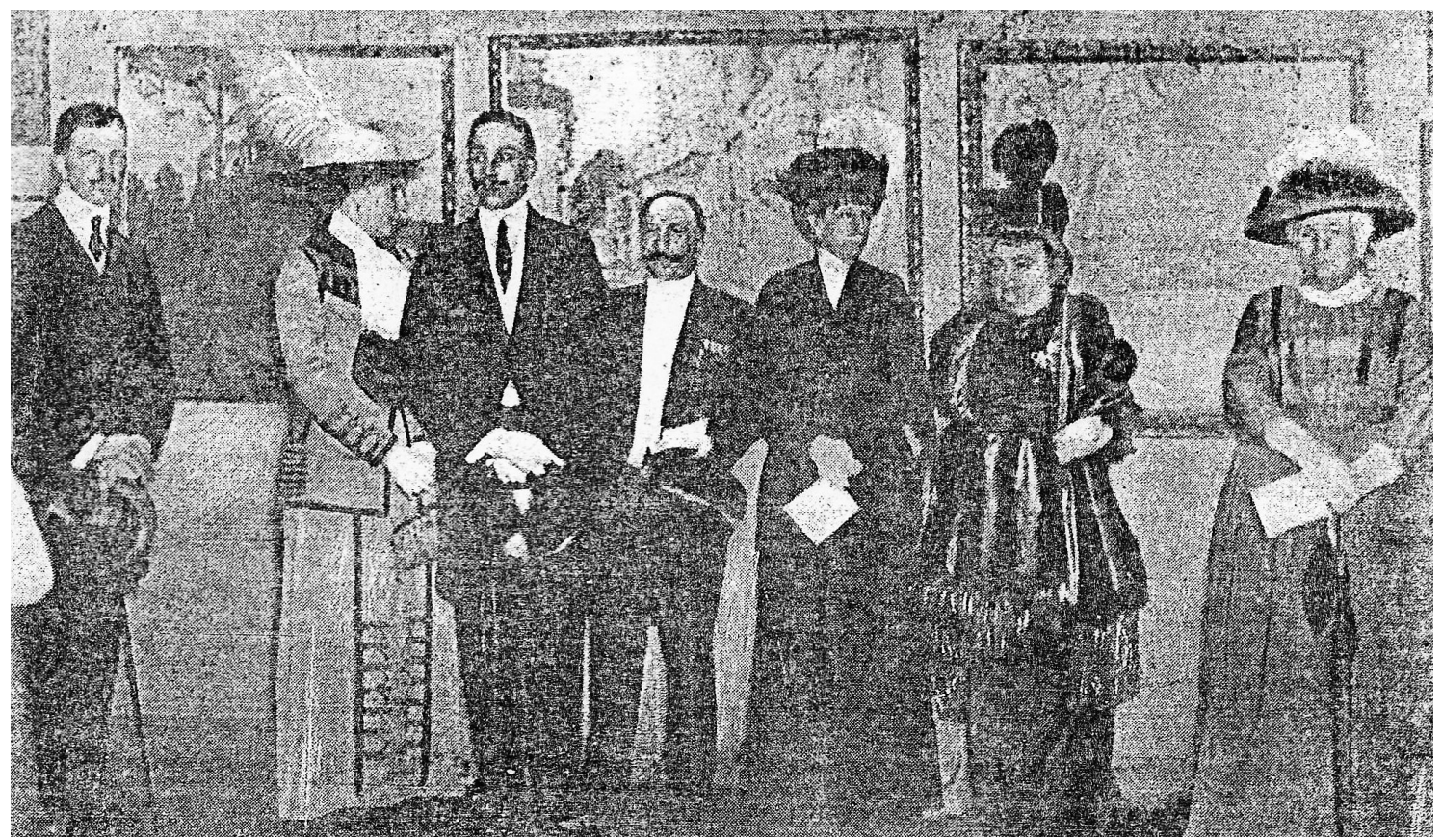

Fig. 4. Fotografía tomada durante la inauguración de la Exposición Regional Gallega de Madrid, 1912 (Pro Galicia, La Habana, núm. 2, p. 23)9

\section{Referencias bibliográficas}

Canitrot Mariño, Prudencio (1908a): “Una visita á la Pardo Bazán”, Galicia (La Habana) 52, pp. 1-2.

— (1908b): "La solidaridad en el campo: Parábolas y promesas", Faro (Madrid) 49, p. 1.

— (1909): "Lo que pasa en Galicia: La próxima revolución”, Faro (Madrid) 50, p. 1.

— (1910): “Una visita á la Pardo Bazán”, en Rías de ensueño. Madrid: Librería de los Sucesores de Hernando, pp. 155-162.

Cansinos Assens, Rafael (1916): “Letras modernísimas. Don Ramón María del Valle-Inclán”, La Correspondencia de España (Madrid) 26-8-1916, p. 3.

- (1995): La novela de un literato. Madrid: Alianza.

Durán, José Antonio (1977): Agrarismo y movilización campesina en el país gallego (1875-1912), Madrid:

Siglo XXI.

Fonte, Ramiro (1985): “Literatos, bohemios e modernistas”, El Correo Gallego 24-3-1985, p. 33.

Gegúndez López, Carlos (2010): “O feito diferencial galego como máximo expoñente da literatura rexional.

A Biblioteca de Escritores Gallegos", Madrygal. Revista de Estudios Gallegos 13, pp. 63-71.

- (2013): Canitrot cen anos de esquecemento. Santiago de Compostela: Alvarellos editora.

- (2014): Canitrot e o sistema literario galego no tránsito de século. Un proxecto didáctico entre o humanismo e a estética galega. Tese de doutoramento. A Coruña: Universidade.

Latorre, Yolanda (1993-94): "El Retablo del Cordero Místico de Van Eyck, símbolo espacio-vital en La Quimera de Emilia Pardo Bazán", Cuadernos de Estudios Gallegos 41/106, pp. 503-510.

Linares Rivas, Manuel (1914): "Prólogo", en VV.AA., La luz apagada, Madrid: Imprenta de Antonio Marzo, pp. 9-10.

Rey Soto, Antonio (1910): "Prudencio Canitrot", en Ruinas, Madrid: Librería de los Sucesores de Hernando, pp. 7-15.

Allí Prudencio Canitrot formaba parte de la comisión organizadora. En este retrato aparece Emilia Pardo Bazán -segunda por la parte derecha-; a su lado, Eduardo Vicenti, alcalde de la capital y representante del Centro Gallego de Madrid, que albergó la muestra artística. También aparecen el rey Alfonso XIII y la reina Victoria junto a otros destacados miembros de la familia real. 